\title{
Neural stem cell degeneration in PD linked to nuclear defects
}

Neural stem cells (NSCs) from patients with Parkinson disease (PD) display ageing-associated nuclear abnormalities that lead to a failure of neurogenesis, according to a recent study in Nature. This study is the first to suggest the involvement of nuclear defects in PD pathogenesis.

"A few years ago we became interested in modelling of ageing-related disease with induced pluripotent stem cells (iPSCs)," says Juan Carlos Izpisua Belmonte, who led the study. "Most studies in PD focus on dopaminergic neurons, but few address the role that NSCs could play, particularly due to the limitations in acquiring these cells."

Speculating that diseases of ageing might be linked to age-dependent alterations in NSCs, the researchers focused their study on the effects of a dominant mutation in leucine-rich repeat kinase 2 (LRRK2), which is associated with both PD pathogenesis and, in adult mice, with impairment of neurogenesis.

First, iPSCs obtained from patients with PD and from healthy controls were differentiated into NSCs in culture. Building on the group's previously established in vitro model of ageing in iPSCs, the cells were then subjected

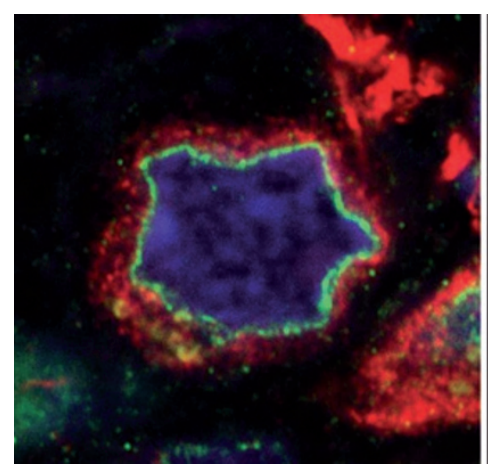

to serial passaging - a process that recapitulates cellular ageing.

"The most striking findings were the age-dependent nuclear aberrations observed in NSCs with the LRRK2 mutation," says Belmonte. Such aberrations were evident in LRRK2mutant cells after 14 rounds of passage but not in control 'aged' cells (wild-type or LRRK2-corrected cells) or in 'young' mutant cells. Late-passage mutant NSCs also had reduced survival compared with controls, and could not be differentiated into neuronal derivatives.

In postmortem human brain samples, immunostaining of the nuclear component lamin B1 revealed altered morphology in the hippocampal dentate gyrus of patients with PD compared with healthy controls.

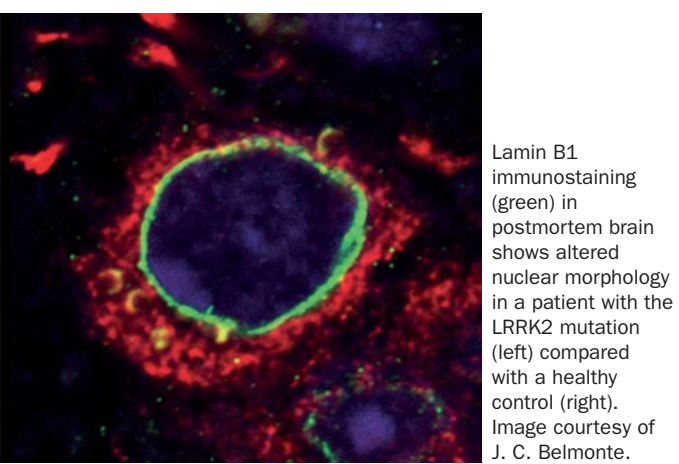

Intriguingly, such nuclear abnormalities were evident not only in patients with the LRRK2 mutation, but also in patients with idiopathic PD.

"Although it is perhaps too early to fully understand the implications of mutant NSCs in PD, we speculate that adult neurogenesis failure might contribute to the nonlocomotor symptoms" concludes Belmonte.Future therapeutic strategies could involve pharmacological targeting of the LRRK2 mutation and re-transplantation of gene-corrected NSCs.

Katy Malpass

Original article Liu, G.-H. et al. Progressive degeneration of human neural stem cells caused by pathogeneic LRRK2. Nature doi:10.1038/nature11557 